OPEN ACCESS

Edited by:

Eva Pontén,

Karolinska Institutet (KI), Sweden

Reviewed by:

Michael James MacLellan,

University of Prince Edward

Island, Canada

Nicolas Alain Turpin,

Université de la Réunion, France

${ }^{*}$ Correspondence: Annike Bekius a.bekius@vu.nl

Specialty section:

This article was submitted to Integrative Physiology, a section of the journal

Frontiers in Physiology

Received: 06 December 2019 Accepted: 18 May 2020

Published: 02 July 2020

Citation:

Bekius A, Bach MM, van der Krogt MM, de Vries R, Buizer Al and Dominici N (2020) Muscle Synergies

During Walking in Children With Cerebral Palsy: A Systematic Review.

Front. Physiol. 11:632.

doi: 10.3389/fphys.2020.00632

\section{Muscle Synergies During Walking in Children With Cerebral Palsy: A Systematic Review}

\author{
Annike Bekius ${ }^{1,2 \star}$, Margit M. Bach ${ }^{1}$, Marjolein M. van der Krogt ${ }^{2}$, Ralph de Vries ${ }^{3}$, \\ Annemieke I. Buizer ${ }^{2}$ and Nadia Dominici ${ }^{1}$
}

${ }^{1}$ Department of Human Movement Sciences, Amsterdam Movement Sciences, Institute of Brain and Behavior Amsterdam, Vrije Universiteit Amsterdam, Amsterdam, Netherlands, ${ }^{2}$ Department of Rehabilitation Medicine, Amsterdam Movement Sciences, Amsterdam UMC, Vrije Universiteit Amsterdam, Amsterdam, Netherlands, ${ }^{3}$ Medical Library, Vrije Universiteit Amsterdam, Amsterdam, Netherlands

Background: Walking problems in children with cerebral palsy $(\mathrm{CP})$ can in part be explained by limited selective motor control. Muscle synergy analysis is increasingly used to quantify altered neuromuscular control during walking. The early brain injury in children with CP may lead to a different development of muscle synergies compared to typically developing (TD) children, which might characterize the abnormal walking patterns.

Objective: The overarching aim of this review is to give an overview of the existing studies investigating muscle synergies during walking in children with CP compared to TD children. The main focus is on how muscle synergies differ between children with $\mathrm{CP}$ and TD children, and we examine the potential of muscle synergies as a measure to quantify and predict treatment outcomes.

Methods: Bibliographic databases were searched by two independent reviewers up to 22 April 2019. Studies were included if the focus was on muscle synergies of the lower limbs during walking, obtained by a matrix factorization algorithm, in children with CP.

Results: The majority $(n=12)$ of the 16 included studies found that children with CP recruited fewer muscle synergies during walking compared to TD children, and several studies $(n=8)$ showed that either the spatial or temporal structure of the muscle synergies differed between children with CP and TD children. Variability within and between subjects was larger in children with CP than in TD children, especially in more involved children. Muscle synergy characteristics before treatments to improve walking function could predict treatment outcomes $(n=3)$. Only minimal changes in synergies were found after treatment.

Conclusions: The findings in this systematic review support the idea that children with CP use a simpler motor control strategy compared to TD children. The use of muscle synergy analysis as a clinical tool to quantify altered neuromuscular control and predict clinical outcomes seems promising. Further investigation on this topic is necessary, and the use of muscle synergies as a target for development of novel therapies in children with CP could be explored.

Keywords: muscle synergy, cerebral palsy, typically developing, children, gait 


\section{INTRODUCTION}

Walking is the most common form of locomotion adopted by humans and limbed animals, and it requires the activation of numerous muscles. It has been theorized, that in order to coordinate this complex behavior, the central nervous system controls basic building blocks, referred to as muscle synergies or motor modules, rather than individual muscles. Muscle synergies are defined as temporal basic activation patterns of different groups of muscles with a corresponding weighting coefficient for every muscle. Each synergy contains multiple muscles and every muscle can contribute to multiple synergies (Ivanenko et al., 2005; Hart and Giszter, 2010; Dominici et al., 2011; Bizzi and Cheung, 2013).

Over the past years, researchers applied muscle synergies as a framework to analyze neuromuscular control in both healthy subjects and individuals with neurological disorders. Generally, muscle synergies are extracted from electromyography (EMG) using matrix factorization algorithms, like the non-negative matrix factorization (NMF), independent component analysis, or factor analysis (Lee and Seung, 1999). In the healthy population, four or five synergies extracted from a large number of EMGs are required during normal walking and these synergies also account for stride-to-stride variability and various speeds (Ivanenko et al., 2004; Cappellini et al., 2006; Clark et al., 2010; Tang et al., 2015). The muscle synergies of healthy adults ("mature synergies") are often used as a template to compare the results of synergy analyses of pathological gait. Muscle synergies appear to be altered in the adult population after brain injury. It has been shown that the number of muscle synergies in post-stroke individuals during walking is reduced compared to unimpaired individuals due to merging of the "mature synergies" observed in healthy adults (Clark et al., 2010). These findings correlate with the degree of motor impairment which might reflect a simplified control strategy of the central nervous system in moderate to severely impaired post-stroke individuals. However, it is unclear whether and how this change in synergy organization can be generalized to other clinical populations and how it relates to gait abnormality.

Cerebral palsy $(\mathrm{CP})$ is a neurodevelopmental motor disorder caused by non-progressive lesions in an immature brain (Himmelmann and Uvebrant, 2018). CP has a wide clinical spectrum, with mobility varying from walking without aids, to being completely wheelchair dependent. The Gross Motor Function Classification System (GMFCS) is used to classify functional mobility in CP. Various diagnostic subtypes exist, based on motor type and distribution of $\mathrm{CP}$, that is, spastic, dyskinetic, or ataxic, and unilateral or bilateral $\mathrm{CP}$, respectively (Bax et al., 2005). Individuals with CP who learn to walk, do so after their brain injury, in contrast to adult stroke survivors who have years of walking experience prior to the brain lesion. In

Abbreviations: BoNT-A, Botulinum Toxin Type A; CP, Cerebral Palsy; EMG, Electromyography; GMFCS, Gross Motor Function Classification System; NMF, Non-Negative Matrix Factorization; SDR, Selective Dorsal Rhizotomy; SEMLS, Single-Event Multilevel Surgery; TD, Typically Developing; VAF, Variance Accounted For; $\mathrm{VAF}_{1}$, Variance Accounted For by one synergy; Walk-DMC, Dynamic Motor Control index during walking. typically developing (TD) children, the number of basic muscle activation patterns increases from two in stepping neonates to four in toddlers, just after their first independent steps (Dominici et al., 2011). The early brain injury in children with CP may lead to a different development of muscle synergies, which might be an underlying factor of abnormal walking patterns. Studies on muscle synergies in children with CP are scarcer than in stroke and have used diverse methods to calculate synergies. Methodological choices in factorization methods, filtering conditions, the number of muscles recorded, and the recording quality appear to influence the outcomes of the synergy calculations (Steele et al., 2013; Santuz et al., 2017; Shuman et al., 2017).

Several types of treatment exist to improve gait quality and functional mobility in children with CP. Recent research has identified the possibility that muscle synergies can predict effectiveness of therapies in children with CP (Schwartz et al., 2016; Shuman et al., 2016, 2018; Oudenhoven et al., 2019). A better insight into the neuromuscular control mechanisms underlying the altered muscle activation patterns in children with $\mathrm{CP}$ could possibly help to improve therapy choices and functional mobility outcomes. In addition, more knowledge about these mechanisms can be important for the interpretation of clinical signs of $\mathrm{CP}$ at an early age, improve indication for therapy in individual patients, and might even be used to develop new diagnostic tools (Cheung et al., 2012).

The present systematic review aims to give an overview of the existing studies investigating muscle synergies in children with $\mathrm{CP}$ during walking to evaluate the current knowledge on this topic. The primary aim is to examine how muscle synergies in children with $\mathrm{CP}$ differ from those exhibited by TD children during walking by investigating the quantification and structure of synergies, and the variability of synergies between and within children with $\mathrm{CP}$. Second, we aim to examine the predictability of treatment outcomes using muscle synergy characteristics, and the effect of treatment on muscle synergies in children with CP.

\section{METHODS}

A systematic review protocol was developed based on the Preferred Reporting Items for Systematic Reviews and MetaAnalyses (PRISMA)-statement (www.prisma-statement.org). It is registered on PROSPERO and can be accessed online (number: CRD42019149109).

\section{Search Strategy}

A comprehensive search was done in the bibliographic databases PubMed, Embase.com, and Web of Science (Core collection), in collaboration with a medical librarian (RV). Databases were searched up to 22 April 2019. The following terms were used including synonyms and closely related words (see Supplementary Information) as index terms or freetext words: "Muscle synergy," "Cerebral palsy," "Typically developing," "Children," "Walking". The search was performed without date, language, or publication status restriction. The full search strategies for all databases can be found in the online Supplementary Information (see Appendix). 


\section{Study Selection}

Studies were eligible for inclusion if they met the following inclusion criteria: (1) children with CP younger than 19 years old, and in case of mixed populations: the majority of the investigated population younger than 19, (2) the focus of the study was on muscle synergies of the lower limb during walking, (3) use of a matrix factorization algorithm to obtain the muscle synergies. Studies were excluded if, (a) it was a conference abstract, (b) it was a conference paper, but a full paper was published afterwards, (c) the study focused on muscle synergies of the upper limb, and (d) the article was a review or protocol.

After exclusion of duplicate articles, two independent reviewers $(\mathrm{AB}$ and $\mathrm{MB})$ performed a title and abstract screening on the residual articles. Thereafter, the reviewers assessed the eligibility of the remaining articles in a full-text screening. Any in- and exclusion conflict among the reviewers was discussed until a consensus was reached. Study designs were defined as being either a cross-sectional, case-control, or retrospective cohort study. Methodological quality and risk of bias of the included articles was assessed using the Downs and Black checklist by the same two independent reviewers (Downs and Black, 1998). In the original scale it is possible to score up to 32 points, but we used a modified version that was applicable for the types of studies included in this systematic review, as has been done in other reviews using the Downs and Black scale (Gorber et al., 2007; Hebert-Losier et al., 2009). This left a maximum total score of 14 points for cross-sectional studies, and 15 points for case-control and cohort studies. Each study was assigned a grade of "excellent" (13-15 points), "good" (10-12 points), "fair" (79 points) or "poor" ( $<7$ points). Any disagreements in grading among the reviewers was discussed until consensus was reached. Articles were not excluded based on poor quality, but this played a role in the overall assessment of the article in the review.

\section{Data Extraction and Analysis}

Data extraction of the included articles was performed independently by $\mathrm{AB}$ and $\mathrm{MB}$. Subject characteristics (age, CP type and distribution, GMFCS), study methods (number of strides analyzed, number of muscles recorded, EMG pre-processing steps, analysis criteria), and outcome measures (muscle synergies) were summarized in a table. The main outcome measures analyzed in this review were: (1) quantification of muscle synergies during walking, such as total number of synergies, $\mathrm{VAF}_{1}$ and walk-DMC, and (2) the spatial and temporal structure of muscle synergies during walking. These outcome measures were assessed in both children with CP and TD children, and pre- and post-treatment in some studies. In addition, variability in number and structure of synergies between and within subjects in the group of children with $\mathrm{CP}$ was evaluated.

\section{RESULTS}

\section{Study Selection}

The electronic search in the cited databases and manual searching of reference lists identified 1127 articles, plus 2 references via additional sources (Figure 1). After duplicate removal, 682 articles were screened on title and abstract, from which 617 were excluded, mostly because of differing target populations (e.g., animals, other diagnosis, or age) or study design (i.e., no muscle synergy analysis during walking involved). Full-text screening of 65 articles left a total of 16 articles that were selected for this review, reasons for exclusion of 49 articles are noted in Figure 1.

\section{Study Characteristics}

Twelve of the 16 articles compared children with CP with TD children, four included only children with CP. The studies varied in sample size, from 3 to 549 children with CP and 8 to 84 TD children. All studies included children with age ranged from 1 to 16 years, in only one study (Steele et al., 2015) older individuals with CP were also included. All studies included children with spastic CP, except for one that included one dyskinetic child (Tang et al., 2015), and GMFCS levels varied from I to IV. An overview of all studies is given in Table $\mathbf{1}$.

\section{Risk of Bias}

Results of the methodological quality assessment are presented in Table 2. Eight studies used a cross-sectional design (Torricelli et al., 2014; Tang et al., 2015; Cappellini et al., 2016, 2018; Goudriaan et al., 2018; Hashiguchi et al., 2018; Kim Y. et al., 2018; Yu et al., 2019), four used case-control designs (Steele et al., 2015, 2019; Shuman et al., 2016, 2017), and four were retrospective cohort studies (Schwartz et al., 2016; Shuman et al., 2018, 2019; Oudenhoven et al., 2019). Quality scores ranged from 5 to 13, one study received the grade "poor" (Torricelli et al., 2014), ten studies "fair" (Tang et al., 2015; Cappellini et al., 2016, 2018; Shuman et al., 2016, 2018, 2019; Hashiguchi et al., 2018; Kim Y. et al., 2018; Steele et al., 2019; Yu et al., 2019), three studies "good" (Steele et al., 2015; Shuman et al., 2017; Goudriaan et al., 2018), and two studies "excellent" (Schwartz et al., 2016; Oudenhoven et al., 2019).

\section{Calculation of Synergies}

All studies used NMF to obtain the muscle synergies from the original (processed) muscle activity. Muscle activity was recorded during overground walking using surface EMG in all cases, 4 to 11 muscles were included per leg, as specified in Table 3. The raw EMG data was most commonly processed using the following steps: high-pass filtered, demeaned (optional), rectified, low-pass filtered, amplitude scaled, and time-normalized. NMF has non-negative constraints, meaning that the original EMG data cannot be negative, and the most used algorithm is the "multiplicative update rule" algorithm presented by Lee and Seung (1999).

\section{Quantification of Synergies}

The quantification of synergies was often done post-hoc based on the variance of EMG activity accounted for (VAF). VAF is a measure of the quality of the EMG reconstruction based on the selected number of muscle synergies. Twelve of the 16 studies included in this review reported the total number of synergies during walking using a certain VAF threshold (Torricelli et al., 2014; Steele et al., 2015, 2019; Tang et al., 2015; Cappellini et al., 2016, 2018; Shuman et al., 2017, 2019; Hashiguchi et al., 2018; 


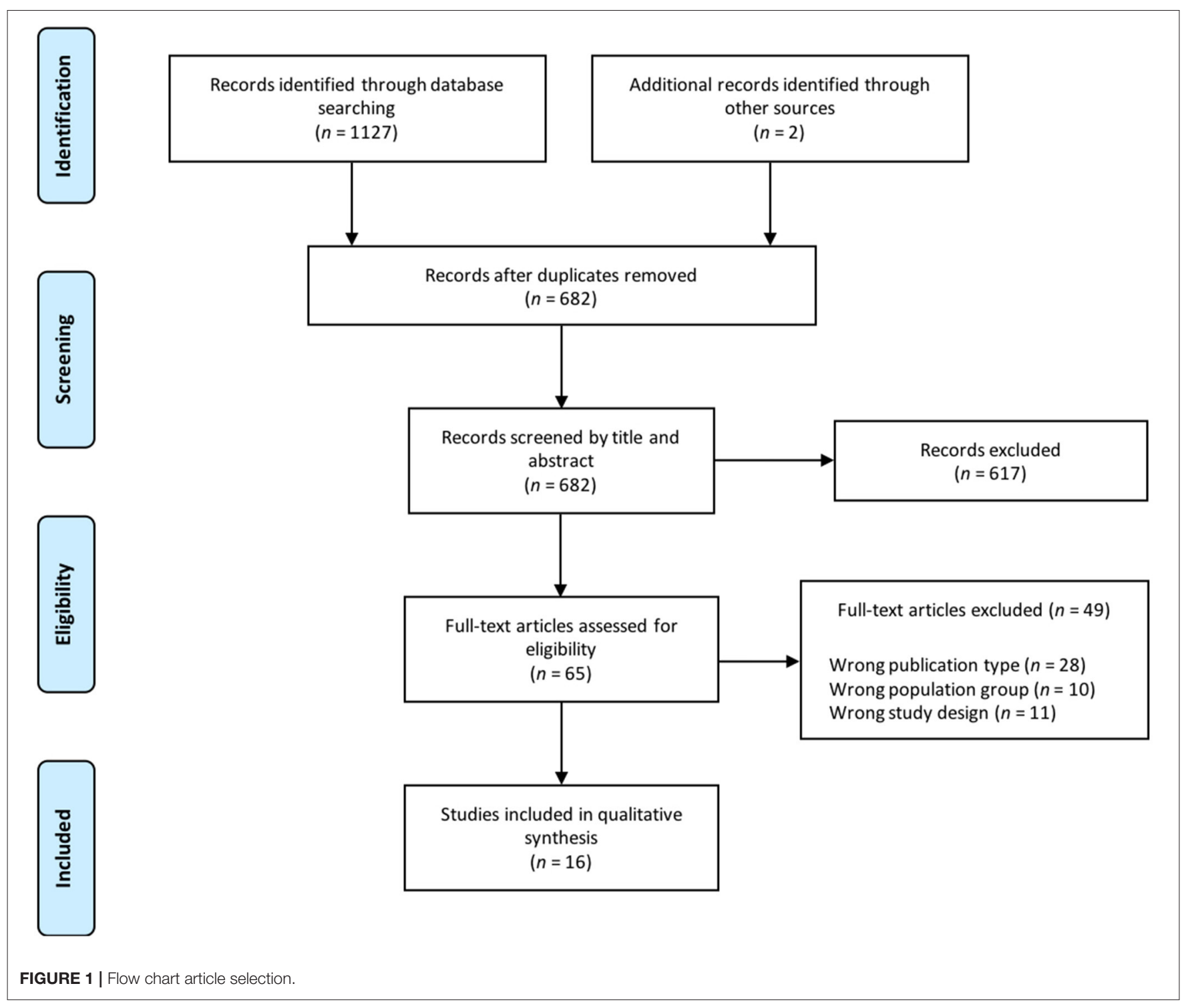

Kim Y. et al., 2018; Oudenhoven et al., 2019; Yu et al., 2019). Out of these articles, nine compared the number of synergies between individuals with $\mathrm{CP}$ and unimpaired individuals (Steele et al., 2015; Tang et al., 2015; Cappellini et al., 2016, 2018; Shuman et al., 2017, 2019; Hashiguchi et al., 2018; Kim Y. et al., 2018; Yu et al., 2019), while the remaining three only included children with CP in their study (Torricelli et al., 2014; Oudenhoven et al., 2019; Steele et al., 2019). Despite a difference in VAF threshold, number of subjects, and number of recorded muscles (see Table 3), the majority of studies found that children with $\mathrm{CP}$ recruited fewer synergies (range 1-4) compared to TD children (range 3-4) or healthy adults (all 4) on average when comparing the number of synergies during walking (Torricelli et al., 2014; Steele et al., 2015; Tang et al., 2015; Shuman et al., 2017; Hashiguchi et al., 2018; Kim Y. et al., 2018; Yu et al., 2019). In contrast, Cappellini et al. $(2016,2018)$ found that both children with CP and TD children recruited 4 synergies. They used a linear regression procedure that plots the VAF against the number of synergies and finds the smallest number for which the root mean square error of the corresponding linear fit is smaller than $10^{-4}$ (d'Avella et al., 2006). The authors show that this corresponds to a VAF $>80 \%$ for all subjects.

Six studies reported $\mathrm{VAF}_{1}$, the variation of EMG activity that can be explained by just one synergy, which is another parameter computed to study the complexity of the locomotor behavior (Steele et al., 2015, 2019; Shuman et al., 2016, 2017; Goudriaan et al., 2018; Kim Y. et al., 2018). Five of the six studies found that the average $\mathrm{VAF}_{1}$ was significantly larger in children with CP (range 71.0-84.2\%) compared to TD children (range 61.0$74.7 \%$, see Table 1). Steele et al. (2019) did not compare with TD children, but showed that $\mathrm{VAF}_{1}$ was $81.4 \pm 5.5 \%$ for children with CP.

Three studies reported the Dynamic Motor Control index during walking (Steele et al., 2015; Shuman et al., 2017; Kim 
TABLE 1 | Summary of the selected study characteristics.

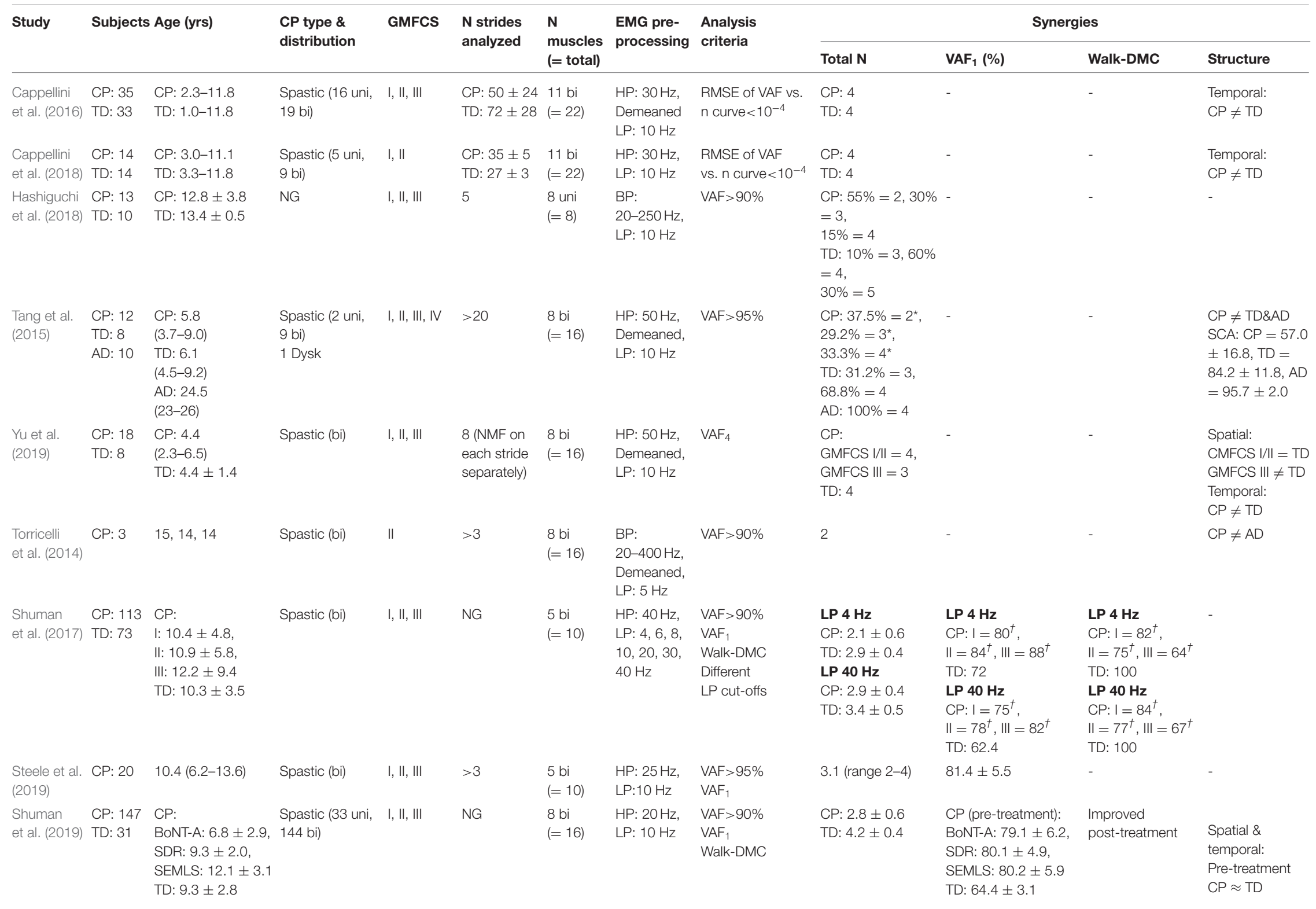


TABLE 1 | Continued

\begin{tabular}{|c|c|c|c|c|c|c|c|c|c|c|c|c|}
\hline \multirow[t]{2}{*}{ Study } & \multirow[t]{2}{*}{ Subjects } & \multirow[t]{2}{*}{ s Age (yrs) } & \multirow{2}{*}{$\begin{array}{l}\text { CP type \& } \\
\text { distribution }\end{array}$} & \multirow[t]{2}{*}{ GMFCS } & \multirow{2}{*}{$\begin{array}{l}\mathrm{N} \text { strides } \\
\text { analyzed }\end{array}$} & \multirow{2}{*}{$\begin{array}{l}\mathrm{N} \\
\text { muscles } \\
(=\text { total })\end{array}$} & \multirow{2}{*}{$\begin{array}{l}\text { EMG pre- } \\
\text { processing }\end{array}$} & \multirow{2}{*}{$\begin{array}{l}\text { Analysis } \\
\text { criteria }\end{array}$} & \multicolumn{4}{|c|}{ Synergies } \\
\hline & & & & & & & & & Total $\mathbf{N}$ & $\mathrm{VAF}_{1}(\%)$ & Walk-DMC & Structure \\
\hline $\begin{array}{l}\text { Oudenhoven } \\
\text { et al. (2019) }\end{array}$ & CP: 36 & $7.2(4-13)$ & Spastic (bi) & I, II, III & 3 & $\begin{array}{l}5 \mathrm{bi} \\
(=10)\end{array}$ & $\begin{array}{l}\mathrm{HP}: 20 \mathrm{~Hz} \\
\text { LP: } 2 \mathrm{~Hz}\end{array}$ & $\mathrm{VAF}>90 \%$ & $\begin{array}{l}\text { Higher } \mathrm{N}=\text { better } \\
\text { treatment outcomes }\end{array}$ & $\begin{array}{l}\text { No correlation with } \\
\text { treatment outcomes }\end{array}$ & - & - \\
\hline $\begin{array}{l}\text { Kim Y. et al. } \\
\text { (2018) }\end{array}$ & $\begin{array}{l}\text { CP: } 20 \\
\text { TD: } 8\end{array}$ & $\begin{array}{l}\text { CP: } 12.5 \pm 3.3 \\
\text { TD: } 12.0 \pm 2.6\end{array}$ & $\begin{array}{l}\text { Spastic (17 uni, } \\
3 \text { bi) }\end{array}$ & I, II & $\begin{array}{l}5 \text { (NMF on } \\
\text { each stride } \\
\text { separately) }\end{array}$ & $\begin{array}{l}8 \mathrm{bi} \\
(=16)\end{array}$ & $\begin{array}{l}\text { HP: } 35 \mathrm{~Hz} \\
\text { LP: } 5 \mathrm{~Hz}\end{array}$ & $\begin{array}{l}\text { VAF }>90 \% \\
V F_{1} \\
\text { Walk-DMC }\end{array}$ & $\begin{array}{l}\text { Mean per stride } \\
\text { CP: } 3.4 \pm 0.3 \\
\text { TD: } 3.8 \pm 0.2\end{array}$ & $\begin{array}{l}\text { CP: } 71 \pm 4 \\
\text { TD: } 61 \pm 3\end{array}$ & $\begin{array}{l}\text { CP: } 65 \pm 14.2 \\
(40.2-91.3) \\
\text { TD: } 100 \pm 10 \\
(85.1-113.0)\end{array}$ & $\begin{array}{l}\text { Spatial: } \\
\text { CP = TD } \\
\text { Temporal: } \\
\text { CP } \neq \text { TD }\end{array}$ \\
\hline $\begin{array}{l}\text { Steele et al. } \\
(2015)\end{array}$ & $\begin{array}{l}\text { CP: } 549 \\
\text { TD: } 84\end{array}$ & $\begin{array}{l}\text { CP: } 9.8 \\
(7.4-13.3)^{\#} \\
\text { TD: } 10.3 \\
(7.6-13.0)^{\#}\end{array}$ & $\begin{array}{l}\text { Spastic (122 uni, } \\
427 \text { bi) }\end{array}$ & I, II, III, IV & 1 & $\begin{array}{l}5 \mathrm{bi} \\
(=10)\end{array}$ & $\begin{array}{l}\text { BP: } \\
20-400 \mathrm{~Hz} \\
\text { LP: } 10 \mathrm{~Hz}\end{array}$ & $\mathrm{VAF}>90 \%$ & $\begin{array}{l}\text { CP: }>80 \%=1 \text { or } 2 \\
\text { TD: }>60 \%=3\end{array}$ & $\begin{array}{l}\text { CP: } 84.2 \\
(83.7-84.7) \\
\text { TD: } 74.6 \\
(71.3-76.1)\end{array}$ & $\begin{array}{l}\text { CP: } 86.2 \\
(85.5-86.9) \\
\text { TD: } 100 \\
(97.9-102.1)\end{array}$ & $\begin{array}{l}\text { Spatial: } \\
\text { CP = TD } \\
\text { Temporal: } \\
\text { CP } \neq \text { TD }\end{array}$ \\
\hline $\begin{array}{l}\text { Shuman } \\
\text { et al. (2016) }\end{array}$ & $\begin{array}{l}\text { CP: } 5 \\
\text { TD: } 6\end{array}$ & $\begin{array}{l}\text { CP: } 10.2 \\
(6.0-13.0) \\
\text { TD: } 10.3 \\
(6.0-13.0)\end{array}$ & $\begin{array}{l}\text { Spastic (2 uni, } \\
3 \text { bi) }\end{array}$ & 1 & $\begin{array}{l}\text { CP: } 47.5 \pm \\
19.6(24-81) \\
\text { TD: } 44.8 \\
\pm 15.9 \\
(25-78)\end{array}$ & $\begin{array}{l}8 \mathrm{bi} \\
(=16)\end{array}$ & $\begin{array}{l}\mathrm{HP}: 40 \mathrm{~Hz} \\
\text { LP: } 4 \mathrm{~Hz}\end{array}$ & $\mathrm{VAF}_{1}$ & - & $\begin{array}{l}\text { CP: } 77.2 \pm 4.1 \\
\text { TD: } 68.4 \pm 2.3\end{array}$ & - & - \\
\hline $\begin{array}{l}\text { Goudriaan } \\
\text { et al. (2018) }\end{array}$ & $\begin{array}{l}\text { CP: } 15 \\
\text { TD: } 15 \\
\text { DMD: } 15\end{array}$ & $\begin{array}{l}\text { CP: } 8.9 \\
(7.6-9.8)^{\#} \\
\text { TD: } 8.6 \\
(7.3-10.0)^{\#} \\
\text { DMD: 8.7 } \\
(6.8-9.9)^{\#}\end{array}$ & $\begin{array}{l}\text { Spastic (8 uni, } \\
7 \text { bi) }\end{array}$ & I, II & 10 & $\begin{array}{l}8 \mathrm{bi} \\
(=16)\end{array}$ & $\begin{array}{l}\text { BP: } \\
20-450 \mathrm{~Hz} \\
\text { LP: } 10 \mathrm{~Hz}\end{array}$ & $\mathrm{VAF}_{1}$ & - & $\begin{array}{l}\text { CP: } 74 \\
\text { TD: } 65 \\
\text { DMD: } 60\end{array}$ & - & - \\
\hline $\begin{array}{l}\text { Schwartz } \\
\text { et al. (2016) }\end{array}$ & CP: 473 & $7.7 \pm 3.3$ & $N G$ & I, II, III & $>4$ & $\begin{array}{l}8 \mathrm{bi} \\
(=16)\end{array}$ & $N G$ & Walk-DMC & - & - & $\begin{array}{l}\text { Higher walk-DMC } \\
\text { pre- treatment = } \\
\text { better outcomes }\end{array}$ & - \\
\hline $\begin{array}{l}\text { Shuman } \\
\text { et al. (2018) }\end{array}$ & $\begin{array}{l}\text { Centre 1 } \\
\text { CP: } 473 \\
\text { TD: } 84 \\
\text { Centre } \mathbf{2} \\
\text { CP: } 163 \\
\text { TD: } 12\end{array}$ & $\begin{array}{l}\text { Centre } 1 \\
\text { CP: } 7.5 \pm 3.4 \\
\text { Centre } \mathbf{2} \\
\text { CP: } 9.3 \pm 2.7\end{array}$ & $N G$ & I, II, III & $N G$ & $\begin{array}{l}\text { Centre 1 } \\
8 \mathrm{bi} \\
(=16) \\
\text { Centre } 2 \\
4 \mathrm{bi} \\
(=8)\end{array}$ & $\begin{array}{l}\text { HP: } 20 \mathrm{~Hz} \\
\text { LP: } 10 \mathrm{~Hz}\end{array}$ & Walk-DMC & - & - & $\begin{array}{l}\mathrm{CP}<\mathrm{TD} \\
\text { Higher walk-DMC } \\
\text { pre- treatment = } \\
\text { better outcomes }\end{array}$ & - \\
\hline
\end{tabular}

CP, cerebral palsy; TD, typically developing; AD, adults; DMD, duchenne muscular dystrophy; Uni, unilateral; Bi, bilateral; Dysk, dyskinetic; GMFCS, gross motor function classification system; N, number of synergies; HP, high-pass filter, LP, low-pass filter; NMF, non-negative matrix factorization; VAF, variance accounted for; VAF synergy comprehensive assessment; NG, not given; BoNT-A, Botulinum Toxin Type A; SDR, Selective Dorsal Rhizotomy; SEMLS, Single-Event Multilevel Surgery. Age and number of strides are given as mean ( 1 standard deviation or the range when provided by the authors) unless marked by a \#, as this signifies the median (25th-75th percentile).

*Values in figure and text are not in agreement, so these values are extracted from the figure; ${ }^{\dagger}$ Signifies that values are extracted from graphical representations and are not precise. 
TABLE 2 | Results of methodological quality assessment.

\begin{tabular}{|c|c|c|c|c|c|c|c|c|c|c|c|c|c|c|c|c|c|c|}
\hline \multirow[t]{2}{*}{ Study } & \multirow[t]{2}{*}{ Study design } & \multicolumn{3}{|c|}{ Reporting } & \multicolumn{2}{|c|}{ Ext. validity } & \multicolumn{4}{|c|}{ Int. validity - bias } & \multicolumn{5}{|c|}{ Int. validity-confounding } & \multirow{2}{*}{$\frac{\text { Power }}{27}$} & \multirow{2}{*}{$\begin{array}{l}\text { Total } \\
\text { score }\end{array}$} & \multirow{2}{*}{ Grade } \\
\hline & & 1234 & 67 & 910 & 11 & 1213 & 141516 & 17 & 18 & 1920 & 21 & 22 & 23 & 24 & $25 \quad 26$ & & & \\
\hline Cappellini et al. (2016) & Cross-sectional & 111 & 11 & 0 & $0^{*}$ & $0^{*}$ & 1 & & 1 & 1 & 1 & $0^{*}$ & & & & 0 & 9 & Fair \\
\hline Goudriaan et al. (2018) & Cross-sectional & 111 & 11 & 0 & $0^{*}$ & $0^{*}$ & 1 & & 1 & 1 & 1 & $0^{*}$ & & & & 1 & 10 & Good \\
\hline Hashiguchi et al. (2018) & Cross-sectional & 111 & 11 & 0 & $0^{*}$ & $0^{*}$ & 1 & & 1 & 1 & 1 & $0^{*}$ & & & & 0 & 9 & Fair \\
\hline Torricelli et al. (2014) & Cross-sectional & 110 & 10 & 0 & 0 & 0 & 1 & & 0 & 1 & $0^{*}$ & $0^{*}$ & & & & 0 & 5 & Poor \\
\hline Yu et al. (2019) & Cross-sectional & 111 & 11 & 1 & $0^{*}$ & $0^{*}$ & 1 & & 1 & 1 & $0^{\star}$ & $0^{*}$ & & & & 0 & 9 & Fair \\
\hline Shuman et al. (2016) & Case-control & 110 & 11 & 1 & $0^{*}$ & $0^{*}$ & 1 & 0 & 1 & 1 & $0^{*}$ & $0^{*}$ & & & & 0 & 8 & Fair \\
\hline Shuman et al. (2017) & Case-control & 111 & 11 & 0 & $0^{*}$ & 1 & 1 & $0^{*}$ & 1 & 1 & 1 & $0^{*}$ & & & & 0 & 10 & Good \\
\hline Shuman et al. (2018) & $\begin{array}{l}\text { Retrospective } \\
\text { cohort }\end{array}$ & 111 & 11 & 0 & 0 & $0^{*}$ & 1 & $0^{*}$ & 1 & 1 & 1 & $0^{*}$ & & & & 0 & 9 & Fair \\
\hline Shuman et al. (2019) & $\begin{array}{l}\text { Retrospective } \\
\text { cohort }\end{array}$ & 110 & 11 & 1 & $0^{*}$ & $0^{*}$ & 1 & $0^{*}$ & 1 & 1 & 1 & $0^{*}$ & & & & 0 & 9 & Fair \\
\hline Oudenhoven et al. (2019) & $\begin{array}{l}\text { Retrospective } \\
\text { cohort }\end{array}$ & 111 & 11 & 0 & 1 & 1 & 1 & 1 & 1 & 1 & 1 & 1 & & & & 0 & 13 & Excellent \\
\hline
\end{tabular}

1: yes; 0:no; O*: unable to determine; Ext.: external; Int.: internal; maximum total scores: cross-sectional = 14; case-control and cohort = 15 .

TABLE 3 | Overview of the recorded and analyzed muscles.

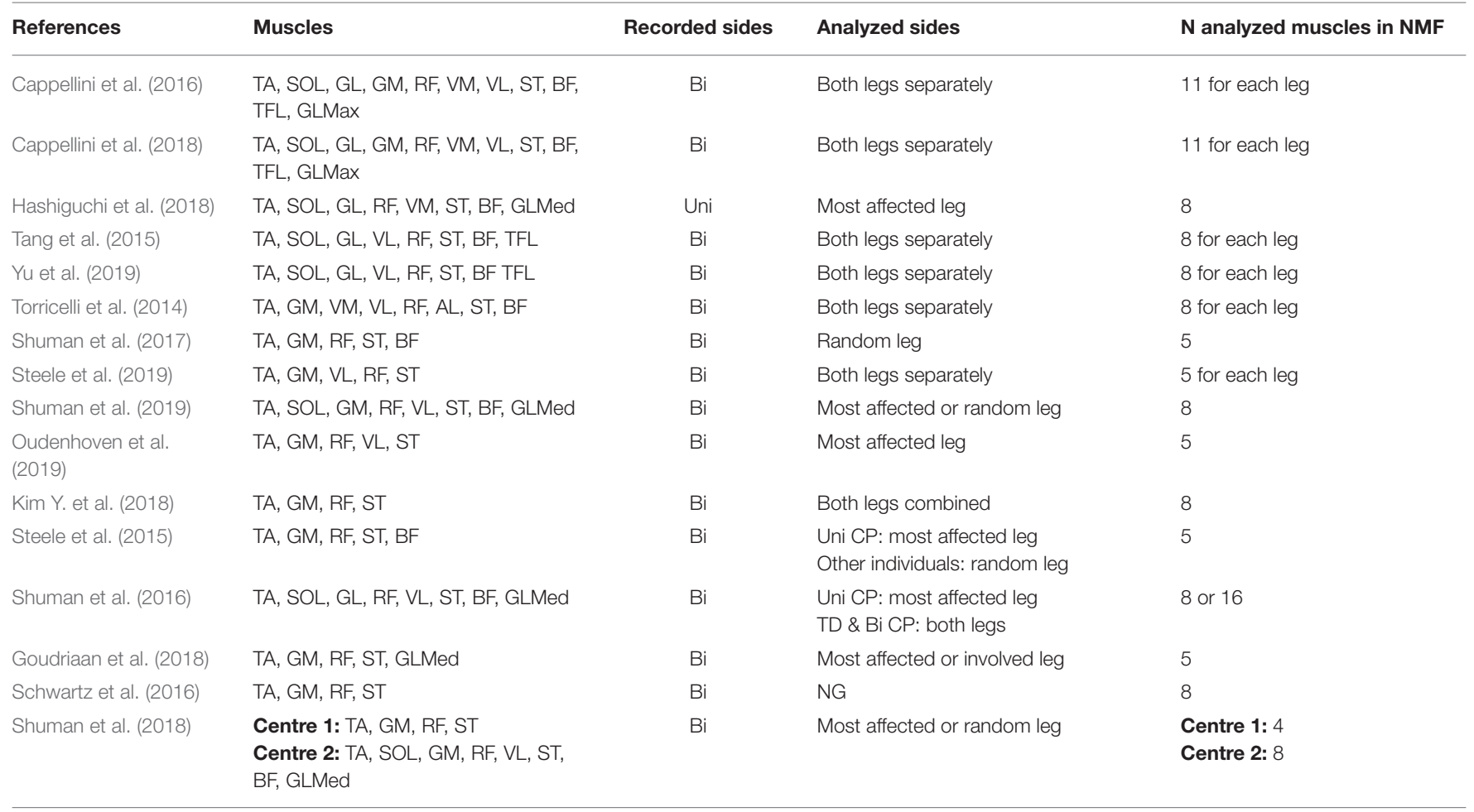

N, number; TA, tibialis anterior; SOL, soleus; GM, gastrocnemius medialis; GL, gastrocnemius lateralis; RF, rectus femoris; VM, vastus medialis; VL, vastus lateralis; ST, semitendinosus; BF, biceps femoris; TFL, tensor fasciae latae; AL, adductor longus; GLMax, gluteus maximus; GLMed, gluteus medius; Uni, unilateral; Bi, bilateral; NMF, non-negative matrix factorization; NG, not given. 
Y. et al., 2018; walk-DMC), which is associated to $\mathrm{VAF}_{1}$, for comparisons of muscle synergies between children with CP and TD children. Walk-DMC transforms $\mathrm{VAF}_{1}$ to a $\mathrm{z}$-score with respect to TD children. A score of 100 signifies the average walkDMC of TD children and each 10-point interval is one standard deviation. Steele et al. (2015) proposed this measure as a clinical tool to quantify altered neuromuscular control, in order to plan treatments and predict clinical outcomes. In agreement with the results on $\mathrm{VAF}_{1}$, all three studies found significantly lower walk-DMC values in children with CP (range of averages 65.086.2) compared to TD children (average 100; Steele et al., 2015; Shuman et al., 2017; Kim Y. et al., 2018). One of these studies showed that an increase in low-pass filter cut-off frequency from 4 to $40 \mathrm{~Hz}$ caused an increase in the total number of synergies, and a decrease in $\mathrm{VAF}_{1}$ in both children with $\mathrm{CP}$ and TD children. However, it had no effect on walk-DMC, since this measure normalizes $\mathrm{VAF}_{1}$ to a $\mathrm{z}$-score (Shuman et al., 2017).

\section{Structure of Synergies}

Eight studies compared the structure of synergies in terms of the results on temporal and spatial patterns between children with CP and controls (Torricelli et al., 2014; Steele et al., 2015; Tang et al., 2015; Cappellini et al., 2016, 2018; Kim Y. et al., 2018; Shuman et al., 2019; Yu et al., 2019). Two studies found that the spatial structure of synergies of children with CP was different from healthy adults ("mature synergies;" Torricelli et al., 2014; Tang et al., 2015), as was assessed by Tang et al. (2015) using a model called synergy comprehensive assessment. In addition, Tang et al. (2015) showed that the spatial structure of synergies in children with CP was different from TD children, and that a large variation in synergy structure was present in the $\mathrm{CP}$ group. The majority of children with $\mathrm{CP}$ showed a combination of "mature synergies" and synergies specific to CP, however none of the affected legs in children with unilateral CP showed merely "mature synergies."

Six studies found that the spatial structure of synergies in both children with $\mathrm{CP}$ and TD children was related to that of "mature synergies," but that the temporal structure differed between children with CP and TD children (Steele et al., 2015; Cappellini et al., 2016, 2018; Kim Y. et al., 2018; Shuman et al., 2019; Yu et al., 2019). These studies found differences in the duration and shifts of the peaks of the temporal patterns within the gait cycle in children with CP compared to TD children. In addition, Yu et al. (2019) showed larger co-activation between synergies and higher variability of the temporal patterns within groups (GMFCS I and II), in children with CP compared to TD children.

\section{Between-Subject Variability}

Five studies discussed the muscle synergy differences within the heterogenous CP group (see Table 4). The relation between the severity of $\mathrm{CP}$ and muscle synergies was examined comparing between different distribution of CP, i.e. uni- or bilateral (Steele et al., 2015; Tang et al., 2015), and levels of impairment of functional mobility, as represented by GMFCS scores and/or Gillette Functional Assessment Questionnaire (Novacheck et al.,
2000) scores (Steele et al., 2015; Tang et al., 2015; Schwartz et al., 2016; Hashiguchi et al., 2018; Kim Y. et al., 2018; Yu et al., 2019).

Children with CP that were bilaterally affected recruited fewer synergies, as identified by lower walk-DMC scores (Steele et al., 2015), a lower total number of synergies, and synergy structures more specific to the CP group (Tang et al., 2015). In addition, higher GMFCS levels in children with $\mathrm{CP}$ were related to lower walk-DMC scores (Steele et al., 2015; Schwartz et al., 2016) and a lower total number of synergies (Tang et al., 2015; Yu et al., 2019). In contrast, Hashiguchi et al. (2018) and Kim Y. et al. (2018) did not find a correlation between the total number of synergies and GMFCS level, although Hashiguchi et al. (2018) found that a higher level of spasticity in children with $\mathrm{CP}$, as assessed by the modified Ashworth Scale, was correlated with a lower number of synergies. The temporal structure of synergies was shown to differ between the affected and less affected side of children with unilateral $\mathrm{CP}$ and children with bilateral $\mathrm{CP}$ (Cappellini et al., 2016), and higher synergy variability was found in children with higher GMFCS levels (Kim Y. et al., 2018; Yu et al., 2019).

\section{Within-Subject Variability}

No systematic differences in number, and spatial or temporal structure of synergies were found between days (Shuman et al., 2016; Steele et al., 2019). However, muscle synergies were found to be variable between strides in both children with CP and TD children (Shuman et al., 2016; Kim Y. et al., 2018). Kim Y. et al. (2018) used a cluster analysis based on a combination of iterative $k$-means clustering and intraclass correlation coefficient analyses to identify stride-to-stride variability of muscle synergies (Kim et al., 2016). The authors found that children with CP had a higher normalized cluster number, meaning that they showed more distinct clusters across strides, although they recruited fewer synergies. Thus, children with CP had higher variability in spatial and temporal synergy structure between strides compared to TD children, for various VAF thresholds (see Table 5).

\section{Treatment}

Three studies investigated whether muscle synergy characteristics in children with CP before treatment are predictive of the effect of different treatments, including selective dorsal rhizotomy (SDR), single-event multilevel orthopedic surgery (SEMLS), single-level orthopedic surgery, botulinum toxin type A (BoNT-A) injection or conservative treatment (physical therapy). Higher walk-DMC values before treatment were associated with improved gait quality, as defined by the Gait Deviation Index and walking speed, after several treatments (Schwartz et al., 2016; Shuman et al., 2018). A higher total number of synergies before treatment was associated with an improved knee angle at initial contact and midstance after SDR, but not with an improvement of overall gait quality, as quantified by the Edinburgh visual gait score (Oudenhoven et al., 2019).

Shuman et al. (2019) investigated whether muscle synergies change after treatment, and whether these changes were associated with treatment outcomes. They found no changes in the number of synergies, or synergy weights, and only minimal 
TABLE 4 | Severity of CP.

\begin{tabular}{|c|c|c|c|c|c|}
\hline References & \multicolumn{2}{|c|}{ Distribution of CP } & \multicolumn{3}{|c|}{ Level of impairment of functional mobility } \\
\hline Tang et al. (2015) & $\begin{array}{l}\text { Uni: } 4 \text { both legs } \\
\text { Bi: } 2 \text { or } 3 \text { per leg }\end{array}$ & - & $\begin{array}{l}\text { GMFCS I: } 2=50 \% ; \\
3=25 \% ; 4=25 \% \\
\text { GMFCS II: } 2=50 \% ; \\
3=17 \% ; 4=33 \% \\
\text { GMFCS III: } 2=25 \% ; \\
3=75 \% \text { GMFCS IV: } 2 \\
=100 \%\end{array}$ & - & - \\
\hline Steele et al. (2015) & - & $\begin{array}{l}\text { He: } 89.2 \text { (87.8-90.6) } \\
\text { Di: } 86.9 \text { (85.9-87.9) } \\
\text { Tri: } 84.4(82.5-86.3) \\
\text { Quad: } 81.4(80.0-82.8)\end{array}$ & - & $\begin{array}{l}\text { GMFCS I: } 92.4 \text { (91.1-93.7) } \\
\text { GMFCS IV: } 79.2(77.5-80.9) \\
\text { FAQ = 10: } 90.9(89.2-92.6) \\
F A Q<7: 80.0(78.7-81.3)\end{array}$ & $\begin{array}{l}\text { Higher GMFCS level = more } \\
\text { synergy structures that are } \\
\text { specific to CP }\end{array}$ \\
\hline Yu et al. (2019) & - & - & $\begin{array}{l}\text { GMFCS I/II = 4; } \\
\text { GMFCS } I I=3\end{array}$ & - & $\begin{array}{l}\text { GMFCS I: DAM = 29.8; } \\
\text { GMFCS II: DAM = 30.6; } \\
\text { TD: DAM = } 26.4\end{array}$ \\
\hline Kim Y. et al. (2018) & - & - & $\begin{array}{l}\text { No correlation with GMFCS } \\
\text { levels }\end{array}$ & - & $\begin{array}{l}\text { GMFCS level was correlated } \\
\text { with normalized cluster } \\
\text { number: } \\
r=0.51, p=0.01\end{array}$ \\
\hline
\end{tabular}

CP, cerebral palsy; N, number of synergies; Walk-DMC, dynamic motor control index during walking; Uni, unilateral; Bi, bilateral; He, hemiplegic; Di, diplegic; Tri, triplegic; Quad, quadriplegic; GMFCS, gross motor function classification system; DAM, deviation of activation matrix (to identify variability of activations patterns between subjects).

TABLE 5 | Variability of synergies.

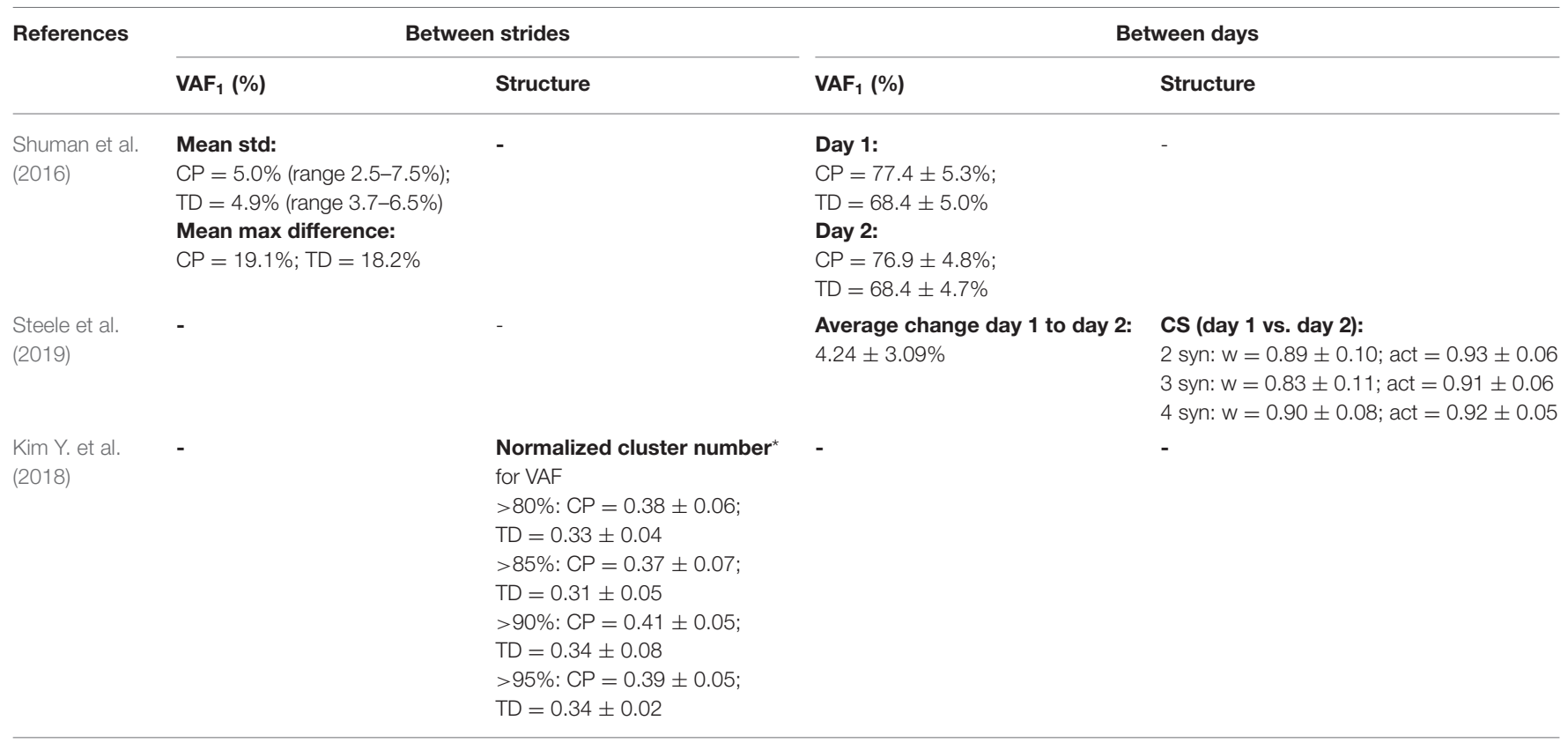

$C P$, cerebral palsy; TD, typically developing; $V A F_{1}$, variance accounted for by one synergy (\%); std, standard deviation; max, maximum; CS, cosine similarity; syn, synergies; $w$, weights; act, activations.

changes in $\mathrm{VAF}_{1}$ after BoNT-A and SDR. Temporal structure of synergies changed only after SDR, toward being more different from TD children. Children with CP whose synergies had a temporal structure more similar to TD children after treatment showed improved gait quality.

\section{DISCUSSION}

Walking problems in children with CP can in part be explained by limited selective motor control, i.e., the impaired ability to use the correct muscle group to move a joint independently 
from other joints in a limb during movement (Desloovere et al., 2006). Muscle synergy analysis is increasingly used to quantify altered neuromuscular control during walking. This systematic review analyzed 16 studies investigating muscle synergies in children with CP during walking, and aimed to examine how these synergies differ from those exhibited by TD children.

\section{Quantification of Synergies}

The majority of studies found that children with CP recruit fewer synergies during walking compared to TD children, either based on a certain VAF threshold, $\mathrm{VAF}_{1}$, or walk-DMC (Torricelli et al., 2014; Steele et al., 2015, 2019; Tang et al., 2015; Schwartz et al., 2016; Shuman et al., 2016, 2017, 2018, 2019; Goudriaan et al., 2018; Hashiguchi et al., 2018; Kim Y. et al., 2018; Yu et al., 2019). The authors of these studies suggest that neuromotor control is altered or less complex in children with CP. The number of synergies for children with $\mathrm{CP}$ and TD children varied between studies. Cappellini et al. were the only ones that did not find a difference in terms of number of synergies between children with CP and TD children (Cappellini et al., 2016, 2018).

The differences in findings between studies may be a consequence of the varying functional mobility levels of subjects included by the different studies. Cappellini et al. (2016, 2018) included children with $\mathrm{CP}$ with a relatively high functional mobility level (77-79\% GMFCS I) compared to the other studies (range 22-67\% GMFCS I), with the exception of Shuman et al. (2016) (100\% GMFCS I). It is plausible that the functional mobility of children with CP and TD children was too similar in Cappellini et al. $(2016,2018)$ to find a difference in the number of synergies between groups.

The use of different methods to define the total number of synergies may also impact synergy outcomes between studies (Hug et al., 2012; Russo et al., 2014). Most studies in this review used VAF to define the total number of synergies, several of these defined a specific VAF threshold, but there is no agreement on the optimal height of this threshold. Consequently, the VAF thresholds ranged from $80 \%$ to $95 \%$ across studies, affecting the total number of synergies that are considered. However, this only influences comparisons of the number of synergies between studies, but differences between groups within one study can still be observed. No systematic differences in number of synergies were found between studies in this review, using different VAF thresholds. To avoid the impact of this threshold, some studies used $\mathrm{VAF}_{1}$ or the related measure walk-DMC, and found results comparable to the VAF threshold. Cappellini et al. $(2016,2018)$ were the only ones using a different method to define the number of synergies, namely the "best linear fit" method. However, it is unlikely that the use of this method explains the similarity in total number of synergies between children with CP and TD children found in Cappellini et al. $(2016,2018)$, since the authors verified that their results agreed with a VAF $>80 \%$. None of the studies in this review considered the added variance of the following synergy as a measure to define the total number of synergies (Clark et al., 2010). The added variance could be an extra tool in the future to define the total number of synergies as it negates the risk that a synergy does not contribute sufficiently to the muscle activation pattern of interest.
The variation in synergy outcomes between studies could also be explained by the different number of muscles recorded. According to previous research, a low number of muscles used for analysis could lead to an over-estimation of VAF (Steele et al., 2013; Zelik et al., 2014; Damiano, 2015). Several studies used NMF to decompose four to eight muscles into two to four synergies, but it is debatable whether this reduction aids enough in terms of easing the interpretation of the data from a statistical point of view. Yet, since it is not feasible to measure all muscles involved in walking, a decomposition will always approximate true neural signaling. Cappellini et al. $(2016,2018)$ were the only ones recording a large number of bilateral muscles, 11 per leg, which may result in a more precise estimation of the muscle synergies involved during walking. This could possibly explain in part why they did not find differences between CP and TD, while others did.

In addition, processing methods of the EMG data, such as filters and amplitude scaling, have been shown to influence muscle synergy outcomes (Shuman et al., 2017). The majority of studies included in this review used a low-pass filter with a cut-off frequency of $10 \mathrm{~Hz}$, but some studies used low-pass filters of $2 \mathrm{~Hz}$ (Oudenhoven et al., 2019), $4 \mathrm{~Hz}$ (Shuman et al., 2016), and $5 \mathrm{~Hz}$ (Torricelli et al., 2014; Kim Y. et al., 2018). The lower the low-pass cut-off frequency, the more data is attenuated, which has been shown to result in a lower number of synergies (van der Krogt et al., 2016; Shuman et al., 2017), and smaller increases in VAF post-treatment (van der Krogt et al., 2016). There is no consensus yet on the best cut-off frequency for a low-pass filter. Different filter types and filter orders are used across studies, but these choices appear to be less significant than the low-pass cut-off frequency (Devaprakash et al., 2016). The influence of methodological choices on muscle synergies is especially important to consider when comparing results across studies or between centers, using different ways to process their data. Overall, despite differences in the number and choice of muscles, and EMG preprocessing methods, studies found similar results. Moreover, the methods were the same in the $\mathrm{CP}$ and TD group within all studies and should therefore have an equal effect on the muscle synergies of all groups. Consequently, these factors are not likely to explain the lack of difference in number of synergies between children with CP and TD children found by Cappellini et al. $(2016,2018)$.

\section{Structure of Synergies}

A subset of the included studies examined differences in the structure of muscle synergies between children with CP and TD children (Torricelli et al., 2014; Steele et al., 2015; Tang et al., 2015; Cappellini et al., 2016, 2018), but they showed different results. Some studies found differences in the spatial structure, i.e., muscle weights, between children with CP and TD children (Torricelli et al., 2014; Tang et al., 2015), whereas others only found differences in the temporal structure, i.e. timing and duration of the peaks of the temporal activation patterns (Torricelli et al., 2014; Steele et al., 2015; Cappellini et al., 2016, 2018; Kim Y. et al., 2018; Yu et al., 2019).

Variation in the use of amplitude scaling methods could result in a different weighting of the synergies per muscle. Scaling 
to unit variance appears to reduce these differences in muscle weights, with more consistent synergy structures across low-pass filters and at a lower number of calculated synergies compared to peak amplitude scaling (Shuman et al., 2017). Although the differences were small, this finding might be specifically interesting for research investigating muscle synergies in clinical populations, which recruit fewer synergies compared to TD children. Moreover, normalization to individual maxima could distort the relative muscle weights due to variable weakness in CP, which can result in inconsistent findings on the spatial structure of synergies across studies (Damiano, 2015).

Deviation from the structure of "mature synergies" in children with CP was found (Torricelli et al., 2014; Tang et al., 2015), and could be a result of the lack of fractionation of synergies, i.e., splitting of one synergy into more, during development. Previous research in stroke patients suggests that a lower number of synergies could result from merging of the synergies of healthy controls (Clark et al., 2010; Cheung et al., 2012). Merging and fractionation of synergies influenced the longitudinal changes of walking patterns in patients after subacute stroke, whereas the number of synergies did not (Cheung et al., 2012; Hashiguchi et al., 2016). Therefore, it might add value to examine the structure including possible fractionation of synergies.

The studies used different methods to quantify similarity between synergy structure. Torricelli et al. (2014) compared the temporal activation patterns using adult data (Winter, 1991), not specifying the method they used, while Tang et al. (2015) and Yu et al. (2019) used Pearson's correlation coefficients, and the other studies used cluster analyses to compare the structure between subjects (Steele et al., 2015; Cappellini et al., 2016, 2018; Kim Y. et al., 2018). These cluster analyses identified comparable patterns across subjects. Three studies isolated the synergies that where not consistent across children as "Not Classified" (Steele et al., 2015; Cappellini et al., 2016, 2018). This means that the synergies that were specific to one child were not considered, and the authors did not quantify how many synergies were removed from each subject. Consequently, differences in synergies within the group of children with $\mathrm{CP}$, and between children with $\mathrm{CP}$ and TD children were possibly lost, which could be a reason why these studies did not find (large) differences in synergy structure between children with CP and TD children. Kim Y. et al. (2018) did allow synergy structures to be assigned to more clusters, and they also found similar synergy structures between children with CP and TD children. However, children with CP recruited fewer synergies per stride, and the use of these structures was less consistent across strides. This means that relative to the number of synergies per stride, children with CP could access more synergy structures than TD children, which suggests that children with CP exhibit the same complexity of synergy structures, but the control of these structures might be decreased. In order to confirm this idea, more studies using the same clustering method are necessary.

Cappellini et al. (2016) found similarities in temporal structure of synergies between children with CP and TD toddlers (1-1.2 years of age) who just started to walk independently. This suggests that muscle synergies in children with CP lag behind in development compared to TD children, which agrees with previous research showing similarity between the walking pattern in children with CP and early gait in TD children (Berger et al., 1982, 1984; Leonard et al., 1991).

\section{Variability of Synergies}

The variation in findings between studies on the number and structure of synergies might be related to the differences in distribution and levels of functional mobility in CP. Children with more severe types of $\mathrm{CP}$, defined by either more distributed CP or higher GMFCS levels, were found to use fewer synergies (Steele et al., 2015; Tang et al., 2015), with different spatial (Tang et al., 2015) and temporal (Steele et al., 2015; Cappellini et al., 2016; Shuman et al., 2019) structures compared to less affected children. These results might reflect a simpler motor control strategy during walking with increasing severity of CP.

In contrast, Hashiguchi et al. (2018) and Kim Y. et al. (2018) did not find a relationship between number of synergies and GMFCS level, possibly because of the small sample size, which limits the variability in a group. Tang et al. (2015) and Yu et al. (2019) also included a limited group of children and they did find an effect of GMFCS level on the number of synergies. Thus, the relationship between the severity of CP and muscle synergies is shown in studies with a sufficient number of subjects (Steele et al., 2015; Cappellini et al., 2016; Shuman et al., 2019), but small sample sizes can coincidentally not show it.

One study found a higher stride-to-stride variability in muscle synergies in children with CP (Kim Y. et al., 2018). This may represent a more immature walking pattern (Hausdorff et al., 1999). High stride-to-stride variability can influence VAF values and thus impact the decomposition of the data into muscle synergies. Only four studies used the minimum of about 20 strides that is necessary according to Oliveira et al. (2014) to create optimal reconstructions of the data and minimize the influence of the variability between strides (Tang et al., 2015; Cappellini et al., 2016, 2018; Shuman et al., 2016). Based on the low amount of studies in this review assessing specifically this aspect we cannot infer whether a lower number of analyzed strides could have an effect on a lower number of synergies.

Considering the high diversity within the group of children with $\mathrm{CP}$, it is not surprising that many studies found larger variability in number and structure of muscle synergies in children with $\mathrm{CP}$ compared to TD children. In some studies children with more severe types of $\mathrm{CP}$ walked with an assistive device or trunk or hand support (Tang et al., 2015; Hashiguchi et al., 2018; Kim Y. et al., 2018; Oudenhoven et al., 2019; Shuman et al., 2019; Yu et al., 2019). In addition, children with more severe types of CP generally walk slower compared to less affected and TD children. Walking speed is an important factor to consider when evaluating muscle synergies, as previous research found that both number and structure of synergies were affected by walking speed in healthy adults (Yokoyama et al., 2016; Kibushi et al., 2018) and TD children (Steele et al., 2015). These findings suggest that different walking speeds require different control from the central nervous system. However, others found that muscle synergies were robust across different walking speeds in healthy adults (Ivanenko et al., 2004; Chvatal and Ting, 2012) and children with CP (Tang et al., 2015; Hashiguchi et al., 2018). 
Although findings are inconsistent, walking speed as a possible confounding factor in comparisons of muscle synergies between children with CP and TD children should be considered during muscle synergy analysis. In addition, the quality of EMG data and the absence of task-independent normalization may have caused variation in muscle synergy results between studies, and should be considered in the future.

\section{Treatment}

The finding that muscle synergies before treatment were correlated with the effect of treatment in children with CP (Shuman et al., 2016, 2018; Oudenhoven et al., 2019), suggests that knowledge about muscle synergies in children with $\mathrm{CP}$ before treatment could help predict whether children will benefit from a specific treatment, and therefore potentially assist in treatment decisions. Walk-DMC has been proposed as a possible measure to quantify altered neuromuscular control pre-treatment, since it has been shown to be correlated with improvement of gait kinematics and walking speed after treatment (Schwartz et al., 2016; Shuman et al., 2018). Importantly, EMG processing methods, and number and type of muscles have limited impact on walk-DMC values. Therefore, this measure could be useful as a comparison of muscle synergy analyses across studies or different clinical centers using different EMG protocols. However, walk-DMC values are highly variable in a heterogeneous population like CP (Steele et al., 2015; Shuman et al., 2018). Although the mean results of walk-DMC values using a large sample size might be a good predictor of treatment outcome, caution should be taken when using individual walk-DMC values in treatment prediction.

Besides the use of muscle synergies as a predictor of treatment outcomes, muscle synergies may also be a target for treatment themselves. Younger children with CP might be more sensitive to interventions (Yang et al., 2013), because their brain is highly plastic and their corticospinal tract is still maturing. Future research should examine the opportunities of specific therapies that target the neural level and adapt muscle synergies, to improve the walking pattern of children with CP. Previous research in unimpaired individuals showed that both the spatial and temporal structure of muscle synergies can change due to intense training in elite athletes (Sawers et al., 2015; Kim M. et al., 2018), and with the use of ankle exoskeletons (Steele et al., 2017; Jacobs et al., 2018). However, current treatments studied in CP were found to have no effect on the spatial structure and merely an effect on the temporal structure of muscle synergies (Shuman et al., 2019). These results suggest that the number and spatial structure of synergies may be hard to change in children with $\mathrm{CP}$, but that the temporal structure of synergies could be a target for treatment. However, normalization of the EMG data is an important factor that may have influenced the results on the spatial structure of synergies. It remains to be further investigated whether novel treatments, such as feedback training (Booth et al., 2019), or therapeutic electrical stimulation of muscles, tendons (Sommerfelt et al., 2001; í et al., 2002; Stackhouse et al., 2007; Wright et al., 2012), or spinal cord (Solopova et al., 2017), could improve muscle synergies, eventually leading to walking improvement.

\section{Future Directions}

The number of studies currently available on this topic is limited, which makes it difficult to draw additional conclusions. With this systematic review we hope to inform researchers about the current research status and to guide them toward better research in the future.

The large variation in number and structure of muscle synergies derived from children with $\mathrm{CP}$ appears to reflect the diversity of $\mathrm{CP}$ and the ability of walking. However, methodological factors also seem to play a role in the determination of muscle synergies. On the one hand, it will be helpful when studies investigating muscle synergies in children with CP use consistent methods across different studies, in order to compare results. On the other hand, this would limit researchers to explore and use novel technologies. At least, researchers could consider recording a number of muscles that is representative for the muscle activation during walking, as well as a sufficient number of strides, in order to make a proper decomposition of muscle synergies. To achieve consistency in EMG data processing steps across studies, researchers should be informed about the choice of filters and factorization methods. The determination of a suitable method to process EMG data of children with CP during walking, for example with a standard EMG processing pipeline, is an important area for future research. If the group of children with $\mathrm{CP}$ is heterogeneous, muscle synergy analysis should be performed on separate groups based for example on different distribution of CP (i.e., unior bilateral $\mathrm{CP}$ ) or different functional mobility levels with sufficient sample sizes, in order to examine the diversity in the CP group. In addition, study of the influence of walking speed on muscle synergies in children with CP and TD children could be useful in the interpretation of the results found in the studies included in this review. Irrespective of the differences in data collection and analysis, the majority of the studies included in this review found similar results, which indicates that the difference in muscle synergies between CP and TD we observe is robust. These corresponding findings from different studies and research groups, provide strong evidence that the observations are related to neural control, and do not merely reflect methodological choices.

It is worth to mention that all the studies reported in this review used the so-called synchronous synergy model (time-invariant synergy approach) to investigate muscle synergies during walking in children with CP. However, various other models such as the time-varying synergy model, first introduced by d'Avella and Tresch (2002), or the space-by-time model (Delis et al., 2014) exist, and could be implemented to study muscle activation modularity in children with CP.

Investigation of the longitudinal development of muscle synergies within subjects would minimize the inter-subject variability and give more insight in the developmental changes in children with CP. Moreover, nothing is known about the development of muscle synergies in very young children at high risk of $\mathrm{CP}$ compared to $\mathrm{TD}$ children. A longitudinal design with consecutive measurements within subjects could give new insights in the development of muscle synergies during walking 
in children with CP, and might open up new paradigms for early interventions in CP.

Despite the increasing number of studies investigating muscle synergies, the underlying mechanisms of muscle synergies remain unknown. It is still a topic of debate whether muscle synergies have a neural or non-neural origin (Bizzi and Cheung, 2013; Zandvoort et al., 2019). Muscle synergies in neonates were shown to mainly reflect spinal cord and brainstem activity, with an increase of the integration of supraspinal and sensory control during development (Dominici et al., 2011). Even though children with CP have cortical lesions, the differences in muscle synergies compared to TD children might also depend on changes in the brainstem and/or spinal cord. In addition, it is debatable whether the use of fewer muscle synergies necessarily reflects less complex motor control, as is suggested in most studies, or whether it is merely caused by higher variability in the EMG data in children with CP. Further research on the underlying mechanisms of muscle synergies is required to answer these questions.

\section{CONCLUSIONS}

In conclusion, the majority of studies found that children with $\mathrm{CP}$ recruit fewer synergies than TD children, and differences in both spatial and temporal structure of synergies were found. In addition, large variability of muscle synergies was found in the group of children with $\mathrm{CP}$, which might be due to the heterogeneity in this group with different functional mobility levels of CP. The inter-subject variability in number and structure of synergies was higher in children with more severe $\mathrm{CP}$, and within subjects the stride-to-stride variability was higher in children with CP compared to TD children, which is known to influence VAF values and thus impact the decomposition of the EMG data into muscle synergies.

The findings in this systematic review support the idea that children with CP use a simpler motor control strategy compared to TD children. The use of muscle synergies as a clinical tool

\section{REFERENCES}

Bax, M., Goldstein, M., Rosenbaum, P., Leviton, A., Paneth, N., Dan, B., et al. (2005). Proposed definition and classification of cerebral palsy, April 2005. Dev. Med. Child Neurol. 47, 571-576. doi: 10.1017/S001216220500112X

Berger, W., Altenmueller, E., and Dietz, V. (1984). Normal and impaired development of children's gait. Hum. Neurobiol. 3, 163-170.

Berger, W., Quintern, J., and Dietz, V. (1982). Pathophysiology of gait in children with cerebral palsy. Electroencephalogr. Clin. Neurophysiol. 53, 538-548. doi: 10.1016/0013-4694(82)90066-9

Bizzi, E., and Cheung, V. C. (2013). The neural origin of muscle synergies. Front. Comput. Neurosci. 7:51. doi: 10.3389/fncom.2013.00051

Booth, A. T. C., van der Krogt, M. M., Harlaar, J., Dominici, N., and Buizer, A. I. (2019). Muscle synergies in response to biofeedback-driven gait adaptations in children with cerebral palsy. Front. Physiol. 10:1208. doi: 10.3389/fphys.2019.01208

Cappellini, G., Ivanenko, Y. P., Martino, G., MacLellan, M. J., Sacco, A., Morelli, D., et al. (2016). Immature spinal locomotor output in children with cerebral palsy. Front. Physiol. 7:478. doi: 10.3389/fphys.2016.00478 to quantify altered neuromuscular control and predict clinical outcomes seems promising. Further investigation on this topic is necessary, and the use of muscle synergies as a target for development of novel therapies in children with CP could be explored.

\section{DATA AVAILABILITY STATEMENT}

The raw data supporting the conclusions of this article will be made available by the authors, without undue reservation, to any qualified researcher.

\section{AUTHOR CONTRIBUTIONS}

$\mathrm{AB}$ and $\mathrm{MB}$ performed the search, quality assessment and data analysis. RV contributed to the search of databases. $\mathrm{AB}$ carried out the drafting of the manuscript. All authors contributed to the manuscript revisions, and approved the submitted version.

\section{FUNDING}

This project has received funding from the European Research Council (ERC) under the European Union's Horizon 2020 research and innovation programme under grant agreement no. 715945 Learn2Walk and from the Dutch Organisation for Scientific Research (NWO) VIDI grant (016.156.346 FirSTeps). The funding sources had no role in study design, data collection and analysis, decision to publish, or preparation of the manuscript.

\section{SUPPLEMENTARY MATERIAL}

The Supplementary Material for this article can be found online at: https://www.frontiersin.org/articles/10.3389/fphys. 2020.00632/full\#supplementary-material
Cappellini, G., Ivanenko, Y. P., Poppele, R. E., and Lacquaniti, F. (2006). Motor patterns in human walking and running. J. Neurophysiol. 95, 3426-3437. doi: 10.1152/jn.00081.2006

Cappellini, G., Sylos-Labini, F., MacLellan, M. J., Sacco, A., Morelli, D., Lacquaniti, F., et al. (2018). Backward walking highlights gait asymmetries in children with cerebral palsy. J. Neurophysiol. 119, 1153-1165. doi: 10.1152/jn.00679.2017

Cheung, V. C., Turolla, A., Agostini, M., Silvoni, S., Bennis, C., Kasi, P., et al. (2012). Muscle synergy patterns as physiological markers of motor cortical damage. Proc. Natl. Acad. Sci. U.S.A. 109, 14652-14656. doi: 10.1073/pnas.1212056109

Chvatal, S. A., and Ting, L. H. (2012). Voluntary and reactive recruitment of locomotor muscle synergies during perturbed walking. J. Neurosci. 32, 12237-12250. doi: 10.1523/JNEUROSCI.6344-11.2012

Clark, D. J., Ting, L. H., Zajac, F. E., Neptune, R. R., and Kautz, S. A. (2010). Merging of healthy motor modules predicts reduced locomotor performance and muscle coordination complexity post-stroke. J. Neurophysiol. 103, 844-857. doi: $10.1152 /$ jn.00825.2009

Damiano, D. (2015). Muscle synergies: input or output variables for neural control? Dev. Med. Child. Neurol. 57, 1091-1092. doi: 10.1111/dmcn.12843 
d'Avella, A., Portone, A., Fernandez, L., and Lacquaniti, F. (2006). Control of fast-reaching movements by muscle synergy combinations. J. Neurosci. 26, 7791-7810. doi: 10.1523/JNEUROSCI.0830-06.2006

d'Avella, A., and Tresch, M. C. (2002). "Modularity in the motor system: decomposition of muscle patterns as combinations of time-varying synergies," in Advances in Neural Information Processing Systems, eds T. G. Dietterich, S. Becker, and Z. Ghahramani (MIT Press), 141-148.

Delis, I., Panzeri, S., Pozzo, T., and Berret, B. (2014). A unifying model of concurrent spatial and temporal modularity in muscle activity. J. Neurophysiol. 111, 675-693. doi: 10.1152/jn.00245.2013

Desloovere, K., Molenaers, G., Feys, H., Huenaerts, C., Callewaert, B., and Van de Walle, P. (2006). Do dynamic and static clinical measurements correlate with gait analysis parameters in children with cerebral palsy? Gait Posture 24, 302-313. doi: 10.1016/j.gaitpost.2005.10.008

Devaprakash, D., Weir, G. J., Dunne, J. J., Alderson, J. A., and Donnelly, C. J. (2016). The influence of digital filter type, amplitude normalisation method, and co-contraction algorithm on clinically relevant surface electromyography data during clinical movement assessments. J. Electromyogr. Kinesiol. 31, 126-135. doi: 10.1016/j.jelekin.2016.10.001

Dominici, N., Ivanenko, Y. P., Cappellini, G., d'Avella, A., Mondi, V., Cicchese, M., et al. (2011). Locomotor primitives in newborn babies and their development. Science 334, 997-999. doi: 10.1126/science.1210617

Downs, S. H., and Black, N. (1998). The feasibility of creating a checklist for the assessment of the methodological quality both of randomised and nonrandomised studies of health care interventions. J. Epidemiol. Commun. Health 52, 377-384. doi: 10.1136/jech.52.6.377

Gorber, S. C., Tremblay, M., Moher, D., and Gorber, B. (2007). A comparison of direct vs. self-report measures for assessing height, weight and body mass index: a systematic review. Obes. Rev. 8, 307-326. doi: 10.1111/j.1467-789X.2007.00347.x

Goudriaan, M., Shuman, B. R., Steele, K. M., Van den Hauwe, M., Goemans, N., Molenaers, G., et al. (2018). Non-neural muscle weakness has limited influence on complexity of motor control during gait. Front. Hum. Neurosci. 12:5. doi: $10.3389 /$ fnhum. 2018.00005

Hart, C. B., and Giszter, S. F. (2010). A neural basis for motor primitives in the spinal cord. J. Neurosci. 30, 1322-1336. doi: 10.1523/JNEUROSCI.5894-08.2010

Hashiguchi, Y., Ohata, K., Kitatani, R., Yamakami, N., Sakuma, K., Osako, S., et al. (2016). Merging and fractionation of muscle synergy indicate the recovery process in patients with hemiplegia: the first study of patients after subacute stroke. Neural Plast 2016:5282957. doi: 10.1155/2016/5282957

Hashiguchi, Y., Ohata, K., Osako, S., Kitatani, R., Aga, Y., Masaki, M., et al. (2018). Number of synergies is dependent on spasticity and gait kinetics in children with cerebral palsy. Pediatr. Phys. Ther. 30, 34-38. doi: 10.1097/PEP.0000000000000460

Hausdorff, J. M., Zemany, L., Peng, C., and Goldberger, A. L. (1999). Maturation of gait dynamics: stride-to-stride variability and its temporal organization in children. J. Appl. Physiol. 86, 1040-1047. doi: 10.1152/jappl.1999.86.3.1040

Hebert-Losier, K., Newsham-West, R. J., Schneiders, A. G., and Sullivan, S. J. (2009). Raising the standards of the calf-raise test: a systematic review. J. Sci. Med. Sport 12, 594-602. doi: 10.1016/j.jsams.2008.12.628

Himmelmann, K., and Uvebrant, P. (2018). The panorama of cerebral palsy in Sweden part XII shows that patterns changed in the birth years 2007-2010. Acta Paediatrica, 107, 462-468. doi: 10.1111/apa.14147

Hug, F., Turpin, N. A., Dorel, S., and Guevel, A. (2012). Smoothing of electromyographic signals can influence the number of extracted muscle synergies. Clin. Neurophysiol. 123, 1895-1896. doi: 10.1016/j.clinph. 2012.01.015

í, Dali, C., Hansen, F. J., Pedersen, S. A., Skov, L., and Hilden, J., Biørnskov, I., et al. (2002). Threshold electrical stimulation (TES) in ambulant children with CP: a randomized double-blind placebo-controlled clinical trial. Dev. Med. Child Neurol. 44, 364-369. doi: 10.1111/j.1469-8749.2002.tb00830.x

Ivanenko, Y. P., Cappellini, G., Dominici, N., Poppele, R. E., and Lacquaniti, F. (2005). Coordination of locomotion with voluntary movements in humans. J. Neurosci. 25, 7238-7253. doi: 10.1523/JNEUROSCI.1327-05.2005

Ivanenko, Y. P., Poppele, R. E., and Lacquaniti, F. (2004). Five basic muscle activation patterns account for muscle activity during human locomotion. J. Physiol. 556, 267-282. doi: 10.1113/jphysiol.2003.057174
Jacobs, D. A., Koller, J. R., Steele, K. M., and Ferris, D. P. (2018). Motor modules during adaptation to walking in a powered ankle exoskeleton. J. Neuroeng. Rehabil. 15:2. doi: 10.1186/s12984-017-0343-x

Kibushi, B., Hagio, S., Moritani, T., and Kouzaki, M. (2018). Speed-dependent modulation of muscle activity based on muscle synergies during treadmill walking. Front. Hum. Neurosci. 12:4. doi: 10.3389/fnhum.2018.00004

Kim, M., Kim, Y., Kim, H., and Yoon, B. (2018). Specific muscle synergies in national elite female ice hockey players in response to unexpected external perturbation. J. Sports Sci. 36, 319-325. doi: 10.1080/02640414.2017.1306090

Kim, Y., Bulea, T. C., and Damiano, D. L. (2016). Novel methods to enhance precision and reliability in muscle synergy identification during walking. Front. Hum. Neurosci. 10:455. doi: 10.3389/fnhum.2016.00455

Kim, Y., Bulea, T. C., and Damiano, D. L. (2018). Children with cerebral palsy have greater stride-to-stride variability of muscle synergies during gait than typically developing children: implications for motor control complexity. Neurorehabil. Neural Repair. 32, 834-844. doi: 10.1177/1545968318796333

Lee, D. D., and Seung, H. S. (1999). Learning the parts of objects by non-negative matrix factorization. Nature 401, 788-791. doi: 10.1038/44565

Leonard, C. T., Hirschfeld, H., and Forssberg, H. (1991). The development of independent walking in children with cerebral palsy. Dev. Med. Child Neurol. 33, 567-577. doi: 10.1111/j.1469-8749.1991.tb14926.x

Novacheck, T. F., Stout, J. L., and Tervo, R. (2000). Reliability and validity of the gillette functional assessment questionnaire as an outcome measure in children with walking disabilities. J. Pediatr. Orthoped. 20, 75-81. doi: 10.1097/01241398-200001000-00017

Oliveira, A. S., Gizzi, L., Farina, D., and Kersting, U. G. (2014). Motor modules of human locomotion: influence of EMG averaging, concatenation, and number of step cycles. Front. Hum. Neurosci. 8:335. doi: 10.3389/fnhum.2014.00335

Oudenhoven, L. M., van der Krogt, M. M., Romei, M., van Schie, P. E. M., van de Pol, L. A., van Ouwerkerk, W. J. R., et al. (2019). Factors associated with longterm improvement of gait after selective dorsal rhizotomy. Arch. Phys. Med. Rehabil. 100, 474-480. doi: 10.1016/j.apmr.2018.06.016

Russo, M., D'Andola, M., Portone, A., Lacquaniti, F., and d'Avella, A. (2014). Dimensionality of joint torques and muscle patterns for reaching. Front. Comput. Neurosci. 8:24. doi: 10.3389/fncom.2014.00024

Santuz, A., Ekizos, A., Janshen, L., Baltzopoulos, V., and Arampatzis, A. (2017). On the methodological implications of extracting muscle synergies from human locomotion. Int. J. Neural Syst. 27:1750007. doi: 10.1142/S0129065717500071

Sawers, A., Allen, J. L., and Ting, L. H. (2015). Long-term training modifies the modular structure and organization of walking balance control. J. Neurophysiol. 114, 3359-3373. doi: 10.1152/jn.00758.2015

Schwartz, M. H., Rozumalski, A., and Steele, K. M. (2016). Dynamic motor control is associated with treatment outcomes for children with cerebral palsy. Dev. Med. Child Neurol. 58, 1139-1145. doi: 10.1111/dmcn.13126

Shuman, B., Goudriaan, M., Bar-On, L., Schwartz, M. H., Desloovere, K., and Steele, K. M. (2016). Repeatability of muscle synergies within and between days for typically developing children and children with cerebral palsy. Gait Posture 45, 127-132. doi: 10.1016/j.gaitpost.2016.01.011

Shuman, B. R., Goudriaan, M., Desloovere, K., Schwartz, M. H., and Steele, K. M. (2018). Associations between muscle synergies and treatment outcomes in cerebral palsy are robust across clinical centers. Arch. Phys. Med. Rehabil. 99, 2175-2182. doi: 10.1016/j.apmr.2018.03.006

Shuman, B. R., Goudriaan, M., Desloovere, K., Schwartz, M. H., and Steele, K. M. (2019). Muscle synergies demonstrate only minimal changes after treatment in cerebral palsy. J. Neuroeng. Rehabil. 16:46. doi: 10.1186/s12984-019-0502-3

Shuman, B. R., Schwartz, M. H., and Steele, K. M. (2017). Electromyography data processing impacts muscle synergies during gait for unimpaired children and children with cerebral palsy. Front. Comput. Neurosci. 11:50. doi: $10.3389 /$ fncom.2017.00050

Solopova, I. A., Sukhotina, I. A., Zhvansky, D. S., Ikoeva, G. A., Vissarionov, S. V., Baindurashvili, A. G., et al. (2017). Effects of spinal cord stimulation on motor functions in children with cerebral palsy. Neurosci. Lett. 639, 192-198. doi: 10.1016/j.neulet.2017.01.003

Sommerfelt, K., Markestad, T., Berg, K., and Saetesdal, I. (2001). Therapeutic electrical stimulation in cerebral palsy: a randomized, controlled, crossover trial. Dev. Med. Child Neurol. 43, 609-613. doi: 10.1017/S0012162201001104

Stackhouse, S. K., Binder-Macleod, S. A., Stackhouse, C. A., McCarthy, J. J., Prosser, L. A., and Lee, S. C. (2007). Neuromuscular electrical stimulation 
versus volitional isometric strength training in children with spastic diplegic cerebral palsy: a preliminary study. Neurorehabil. Neural Repair. 21, 475-485. doi: $10.1177 / 1545968306298932$

Steele, K. M., Jackson, R. W., Shuman, B. R., and Collins, S. H. (2017). Muscle recruitment and coordination with an ankle exoskeleton. J. Biomech. 59, 50-58. doi: 10.1016/j.jbiomech.2017.05.010

Steele, K. M., Munger, M. E., Peters, K. M., Shuman, B. R., and Schwartz, M. H. (2019). Repeatability of electromyography recordings and muscle synergies during gait among children with cerebral palsy. Gait Posture 67, 290-295. doi: 10.1016/j.gaitpost.2018.10.009

Steele, K. M., Rozumalski, A., and Schwartz, M. H. (2015). Muscle synergies and complexity of neuromuscular control during gait in cerebral palsy. Dev. Med. Child Neurol. 57, 1176-1182. doi: 10.1111/dmcn.12826

Steele, K. M., Tresch, M. C., and Perreault, E. J. (2013). The number and choice of muscles impact the results of muscle synergy analyses. Front. Comput. Neurosci. 7:105. doi: 10.3389/fncom.2013.00105

Tang, L., Li, F., Cao, S., Zhang, X., Wu, D., and Chen, X. (2015). Muscle synergy analysis in children with cerebral palsy. J. Neural Eng. 12:046017. doi: 10.1088/1741-2560/12/4/046017

Torricelli, D., Pajaro, M., Lerma, S., Marquez, E., Martinez, I., Barroso, F., et al. (2014). "Modular control of crouch gait in spastic cerebral palsy," in XIII Mediterranean Conference on Medical and Biological Engineering and Computing 2013 (Springer), 1718-1721. doi: 10.1007/978-3-319-00846-2_424

van der Krogt, M. M., Oudenhoven, L., Buizer, A. I., Dallmeijer, A., Dominici, N., and Harlaar, J. (2016). The effect of EMG processing choices on muscle synergies before and after BoNT-A treatment in cerebral palsy. Gait Posture 49:31. doi: 10.1016/j.gaitpost.2016.07.095

Winter, D. A. (1991). Biomechanics and Motor Control of Human Gait: Normal, Elderly and Pathological. Waterloo: University of Waterloo Press.

Wright, P. A., Durham, S., Ewins, D. J., and Swain, I. D. (2012). Neuromuscular electrical stimulation for children with cerebral palsy: a review. Arch. Dis. Child 97, 364-371. doi: 10.1136/archdischild-2011-3 00437

Yang, J. F., Livingstone, D., Brunton, K., Kim, D., Lopetinsky, B., Roy, F., et al. (2013). Training to enhance walking in children with cerebral palsy: are we missing the window of opportunity? Semin. Pediatr. Neurol. 20, 106-115. doi: 10.1016/j.spen.2013.06.011

Yokoyama, H., Ogawa, T., Kawashima, N., Shinya, M., and Nakazawa, K. (2016). Distinct sets of locomotor modules control the speed and modes of human locomotion. Sci. Rep. 6:36275. doi: 10.1038/srep36275

Yu, Y., Chen, X., Cao, S., Wu, D., Zhang, X., and Chen, X. (2019). Gait synergetic neuromuscular control in children with cerebral palsy at different gross motor function classification system levels. J. Neurophysiol. 121, 1680-1691. doi: 10.1152/jn.00580.2018

Zandvoort, C. S., van Dieen, J. H., Dominici, N., and Daffertshofer, A. (2019). The human sensorimotor cortex fosters muscle synergies through cortico-synergy coherence. Neuroimage 199, 30-37. doi: 10.1016/j.neuroimage.2019.05.041

Zelik, K. E., La Scaleia, V., Ivanenko, Y. P., and Lacquaniti, F. (2014). Can modular strategies simplify neural control of multidirectional human locomotion? J. Neurophysiol. 111, 1686-1702. doi: 10.1152/jn.00776.2013

Conflict of Interest: The authors declare that the research was conducted in the absence of any commercial or financial relationships that could be construed as a potential conflict of interest.

Copyright (C) 2020 Bekius, Bach, van der Krogt, de Vries, Buizer and Dominici. This is an open-access article distributed under the terms of the Creative Commons Attribution License (CC BY). The use, distribution or reproduction in other forums is permitted, provided the original author(s) and the copyright owner(s) are credited and that the original publication in this journal is cited, in accordance with accepted academic practice. No use, distribution or reproduction is permitted which does not comply with these terms. 